\title{
Influência da Concentração e do Tempo de Condicionamento com Ácido Hidrofluorídrico na Rugosidade e Morfologia Superficial de uma Zircônia Glazeada
}

\section{Influence of Hydrofluoric Acid Concentration and Etching Time on the Surface Roughness and Morphology of a Glazed Zirconia}

\author{
FERNANDA CAMPOS ${ }^{1}$ \\ SARINA MACIEL BRAGA PEREIRA \\ SABRINAALVES FEITOSA ${ }^{2}$ \\ AMANDA MARIA DE OLIVEIRA DAL PIVA ${ }^{3}$ \\ LUIZ FELIPE VALANDRO 4 \\ MARCO ANTONIO BOTTINO 5 \\ RODRIGO OTHÁVIO DE ASSUNÇÃO E SOUZA 6
}

RESUMO

Objetivo: Avaliar o efeito da concentração e do tempo de condicionamento com ácido fluorídrico na rugosidade e morfologia superficial de uma zircônia glazeada. Materiais e Métodos: Blocos de cerâmica à base de zircônia $(5 \times 5 \times 3 \mathrm{~mm}$ ) (Vita YZ-2000-Cubes, VitaZahnfabrik, Alemanha) foram confeccionados e divididos em 6 grupos, de acordo com os fatores "concentração do ácido fluorídrico (HF)" ( $5 \%$ e $10 \%$ ) e "tempo de condicionamento" (20 s, 60 s e $90 \mathrm{~s})(\mathrm{n}=2)$ : HF $10 / 20=$ HF $10 \%+20 \mathrm{~s} ; \mathrm{HF} 10 / 60=\mathrm{HF} 10 \%+60 \mathrm{~s} ; \mathrm{HF} 10 / 90=\mathrm{HF}$ $10 \%+90 \mathrm{~s} ; \mathrm{HF} 5 / 20=\mathrm{HF} 5 \%+20 \mathrm{~s} ; \mathrm{HF} 5 / 60=\mathrm{HF} 5 \%+60 \mathrm{~s} ; \mathrm{HF} 5 /$ $90=$ HF $5 \%+90 \mathrm{~s}$. O glaze (Vita Akzent, Vita-Zahnfabrik, Alemanha) foi aplicado com o auxílio de um pincel e sinterizado de acordo com as recomendações do fabricante. Após os diferentes protocolos de condicionamento, foram aferidas cinco medições de rugosidade (Ra) para cada espécime em Perfilômetro Digital (Wyko ${ }^{\circledR}$, Modelo NT1100 , Veeco, EUA). Os dados ( $\mu \mathrm{m})$ obtidos foram analisados estatisticamente com análise de variância (ANOVA 2-fatores) e Teste de Tukey (95\%). Resultados: O fator "concentração do ácido fluorídrico" $(p=0,149)$ não foi significante estatísticamente. No entanto, o fator "tempo de condicionamento" ( $p=0,009)$ foi significante. A interação entre os fatores também apresentou significância estatística $(p=0,00)$. As médias de rugosidade ( \pm desvio-padrão) obtidas foram $(\mu \mathrm{m})$ : GHF $10 / 20=1,94( \pm 0,72)^{\mathrm{A}}, \quad \operatorname{GHF} 5 / 90=1,92( \pm 0,19)^{\mathrm{A}}, \quad \mathrm{GHF} 5 /$ $20=1,38( \pm 0.48)^{\mathrm{AB}}, \mathrm{GHF} 5 / 60=1,18( \pm 0,63)^{\mathrm{B}}, \mathrm{GHF} 10 / 60=1,17( \pm 0,30)^{\mathrm{B}}$, GHF10/90 $=0,82( \pm 0,27)^{\mathrm{B}}$ (Tukey). Conclusão: Conclui-se que o ácido fluorídrico $(10 \%)$ em maior concentração, associado a um menor tempo de condicionamento (20 s), promoveu maior rugosidade superficial da zircônia glazeada.

DESCRITORES

Cerâmica. Zircônio. Vitrificação.

\begin{abstract}
Objective: The aim of this study was to evaluate the effect of different etching protocols on the surface roughness of a glazed zirconia. Material and Methods: Zirconia ceramic-blocks (5 $55 \times 3 \mathrm{~mm}$ ) (Vita YZ-2000-Cubes, Vita-Zahnfabrik, Germany) were prepared and divided into 6 groups according to the variables "concentration of hydrofluoric acid (HF)" (5\% and $10 \%)$ and "etching time " (20 s, $60 \mathrm{~s}$ and $90 \mathrm{~s})$ (n = 2), as follows: HF10/20 = HF $10 \%+20 \mathrm{~s} ; \mathrm{HF} 10 / 60=\mathrm{HF} 10 \%+60$ s; $\mathrm{HF} 10 / 90=\mathrm{HF} 10 \%+90 \mathrm{~s} ; \mathrm{HF} 5 / 20=\mathrm{HF} 5 \%+20 \mathrm{~s} ; \mathrm{HF} 5 / 60=\mathrm{HF}$ $5 \%+60 \mathrm{~s} ; \mathrm{HF} 5 / 90=\mathrm{HF} 5 \%+90 \mathrm{~s}$. The glaze (Vita Akzent, VitaZahnfabrik, Germany) was applied with a brush and sintered according to the manufacturer's recommendations. After different etching protocols, five roughness $(\mathrm{Ra})$ measurements were taken for each specimen in a digital profilometer (Wyko $\AA$, Model NT-1100, Veeco, USA). The data were statistically analyzed using analysis of variance (ANOVA 2-way) and Tukey's test (95\%). Results: The variable "concentration of hydrofluoric acid" $(p=0.149)$ was not statistically significant, while the variable "etching time" $(p=0.009)$ was significant. The interaction between variables was statistically significant $(p=$ $0.00)$. The roughness averages ( \pm standard deviation) obtained were $(\mu \mathrm{m}): \mathrm{GHF} 10 / 20=1.94( \pm 0.72)^{\mathrm{A}}, \mathrm{GHF} 5 / 90=1.92( \pm 0.19)^{\mathrm{A}}, \mathrm{GHF} 5 /$ $20=1.38( \pm 0.48)^{\mathrm{AB}}, \mathrm{GHF} 5 / 60=1.18( \pm 0.63)^{\mathrm{B}}, \mathrm{GHF} 10 / 60=1.17( \pm 0.30)^{\mathrm{B}}$, GHF10/90 $=0.82( \pm 0.27)^{\mathrm{B}}$ (Tukey). Conclusion: It can be concluded that hydrofluoric acid $(10 \%)$ at a higher concentration associated with a lower etching time (20 s) promoted higher superficial roughness on glazed zirconia.
\end{abstract}

DESCRIPTORS

Ceramics, Zirconium Vitrification.

Professora Doutora da Disciplina de Prótese do Centro Universitário de João Pessoa/Unipê- João Pessoa, Paraĺba, Brasil.

2 Doutora em Odontologia Restauradora. José dos Campos, São Paulo, Brasil.

3 Mestranda em Odontologia Restauradora. Instituto de Ciência e Tecnologia, Universidade Estadual Paulista “Júlio de Mesquita Filho"/UNESP, São José dos Campos, São Paulo, Brasil.

4 Professor Adjunto Doutor da Disciplina de Prótese Fixa do Departamento de Odontologia Restauradora, Universidade Federal de Santa Maria/UFSMSanta Maria, Rio Grande do Sul, Brasil.

5 Professor Titular Doutor do Instituto de Ciência e Tecnologia, Universidade Estadual Paulista “Júlio de Mesquita Filho"/UNESP- São José dos Campos, São Paulo, Brasil.

6 Professor Adjunto Doutor Departamento de Odontologia Restauradora, Universidade Federal do Rio Grande do Norte/UFRN- Natal, Brasil. 
A cerâmica de zircônia estabilizada por itria (Y-TZP) tem se tornado popular como infraestrutura de coroas e próteses parciais fixas devido a fatores como a sua biocompatibilidade e excelentes propriedades mecânicas, somados ao avanço da tecnologia CAD/ CAM (Computer Aided Design - Computer Aided machining) $]^{1,2,3}$. No entanto, estudos clínicos têm mostrado que falhas por perda de retenção (descimentação) estão entre as mais frequentes quando se utilizam restaurações com infraestrutura de zircônia, levando à necessidade de sua recimentação $0^{4,5}$.

Esta perda de retenção pode acontecer em decorrência da baixa adesão entre zircônia e cimento resinoso, pois este material sofre pouca ou nenhuma degradação superficial pela ação do ácido fluorídrico (HF) ${ }^{6}$. Neste contexto, diversos estudos têm procurado modificar a superfície dessa cerâmica para melhorar a união desta com o cimento resinoso $0^{7,8,9}$. Um tratamento de superfície bastante utilizado é o jateamento com partículas de alumina e partículas de alumina revestidas por sílica $^{8}$. Este jateamento gera um aumento da rugosidade superficial, fazendo com que a união micromecânica com o cimento resinoso seja promovida ${ }^{10}$. Além disso, o jateamento com partículas revestidas por sílica promove a deposição de sílica na superfície cerâmica (silicatização), o que aumentaria ainda mais a união entre zircônia e cimento resinoso ${ }^{11,12}$. Entretanto, apesar de gerar resultados satisfatórios com relação à resistência de união, o jateamento pode gerar microtrincas na superfície da zircônia devido à pressão e velocidade com que as partículas são lançadas ${ }^{13,14}$.

Outra técnica que tem sido estudada, envolve a aplicação de uma fina camada de glaze na superfície interna da restauração previamente à cimentação $0^{2,6,15,16}$. Este tratamento permite que o condicionamento ácido e a silanização, procedimentos geralmente utilizados durante a cimentação convencional de cerâmicas vítreas, sejam realizados ${ }^{16}$. Em cerâmicas ácido-sensíveis, o condicionamento ácido aumenta a rugosidade superficial devido à dissolução seletiva da fase vítrea presente em grande quantidade neste material ${ }^{17}$. Adicionalmente, o condicionamento ácido aumenta a molhabilidade e a energia de superfície destas cerâmicas ${ }^{18}$.

Os estudos que propõem técnicas de deposição de camadas vítreas para posterior condicionamento com ácido fluorídrico mostram protocolos de condicionamento diversos ${ }^{2,6,15,16,19}$. Os protocolos utilizados geralmente utilizam as recomendações para cerâmicas ácido-sensíveis, que não são específicas para superfícies de zircônia glazeada. O ácido fluorídrico tem sido utilizado em diferentes concentrações e tempos de condicionamento, resultando em resultados controversos $2,16,19$ utilizaram ácido fluorídrico com concentrações de 9 a $10 \%$, durante 20,60 ou 90 segundos, obtendo valores de resistência de união maiores do que quando utilizaram jateamento de partículas de alumina e de alumina revestidas por sílica. Já em um estudo ${ }^{15}$ recente, foi utilizado ácido fluorídrico a $9,5 \%$ por dois minutos, concluindo que a cobertura da superfície com uma camada de glaze é uma técnica promissora. Resultados semelhantes também tem sido relatado pela literatura ${ }^{7}$ quando se comparou o glazeamento da zircônia com a aplicação de primer.

Considerando a importância de adequar essa nova técnica aos protocolos de cimentação já estabelecidos pela literatura (ácido fluorídrico + agente de união silano), este trabalho se propõe a avaliar o efeito de diferentes protocolos de condicionamento na rugosidade superficial de uma zircônia glazeada na superfície de cimentação. A hipótese testada foi de que os protocolos de condicionamento resultarão em valores de rugosidade diferentes.

\section{MATERIAIS E MÉTODOS}

Os materiais utilizados neste estudo, bem como a respectiva marca comercial e fabricante estão apresentados no Quadro 1.

\section{Confecção dos espécimes}

Inicialmente, blocos de zircônia foram cortados em máquina de corte (Isomet, Buehler, Estados Unidos) nas dimensões $7 \mathrm{~mm}(\mathrm{~L}) \times 7 \mathrm{~mm}$ (P) $\times 3,5 \mathrm{~mm}$ (A). Em seguida, foram lixados com lixas d'água $\left(n^{\circ} 1200\right)$ para regularização das superfícies. Após lavagem em cuba ultrassônica com água destilada por 5 minutos e secagem, os blocos foram levados ao forno Zyrcomat (Vita Zahnfabrik, Alemanha) para sinterização de acordo com ciclo de queima recomendado pelo fabricante (Figura 1A).

\section{Aplicação do glaze}

O glaze VitaAkzent (Vita Zahnfabrik, Alemanha) foi manipulado de acordo com as 


\begin{tabular}{ccc}
\hline $\begin{array}{l}\text { Quadro 1: Nomes comerciais, tipos de material e fabricantes dos produtos a serem } \\
\text { utilizados nos experimentos. } \\
\text { Nome comercial }\end{array}$ & Tipo de material & Fabricante \\
InCeram YZ 2000 Cubes & Cerâmica a base de zircônia & Vita Zahnfabrik, Alemanha \\
Glaze Akzent & Glaze & Vita Zahnfabrik, Alemanha \\
$\begin{array}{c}\text { Condicionador de } \\
\text { porcelana } \\
-\end{array}$ & Ácido Flurídrico 10\% & Dentsply, Brasil \\
& Ácido Flurídrico 5\% & $\begin{array}{c}\text { Fórmula e Ação - Farmácia } \\
\text { de Manipulacão, Brasil }\end{array}$ \\
\hline
\end{tabular}

recomendações do fabricante e aplicado com o auxílio de um pincel sobre a superfície dos blocos de zircônia (Figura 1B). Em seguida, os blocos foram levados a forno específico (Vita Vacumat $6000 \mathrm{MP}$, Vita Zahnfabrik, Alemanha) para queima do glaze segundo recomendações do fabricante (Figura 1C).

Os blocos de zircônia glazeada foram divididos em 6 grupos $(n=10)$, de acordo com os fatores "concentração do ácido fluorídrico" (10 \% e $5 \%$ ) e "tempo de condicionamento" (20 s, $60 \mathrm{~s} \mathrm{e}$ 90 s): HF10/20 = ácido fluorídrico $10 \%+20$ s de condicionamento; HF10/60 = ácido fluorídrico 10\% + $60 \mathrm{~s}$ de condicionamento; HF10/90 = ácido fluorídrico $10 \%+90$ s de condicionamento; HF5/ 20 = ácido fluorídrico $5 \%+20 \mathrm{~s}$ de condicionamento; HF5/60 = ácido fluorídrico $5 \%+60 \mathrm{~s}$ de condicionamento; HF5/90 = ácido fluorídrico $5 \%$ +90 s de condicionamento.

\section{Condicionamento da superfície}

As superfícies glazeadas foram condicionadas com ácido fluorídrico (HF) na concentração de $10 \%$ e $5 \%$ por tempos variados (20 s, $60 \mathrm{~s}$ e $90 \mathrm{~s})$. Após, as superfícies foram lavadas com jato de água por $30 \mathrm{~s}$ e secas com jatos de ar. Em seguida, as amostras foram lavadas em ultrassom por 5 min em água destilada e secas com jatos de ar.

\section{Análise do padrão topográfico da superfície condicionada}

Para análise quantitativa da geometria tridimensional (3D) da superfície da zircônia após condicionamento do glaze, as amostras foram analisadas em perfilômetro óptico digital $\left(\mathrm{Wyko}^{\circledR}\right.$, Modelo NT 1100, Veeco, EUA). Este perfilômetro é conectado a uma unidade computadorizada contendo o programa Wyko Vision 32 (Wyko®, Veeco, EUA) para análise dos dados. As medições dos parâmetros foram realizadas com um aumento
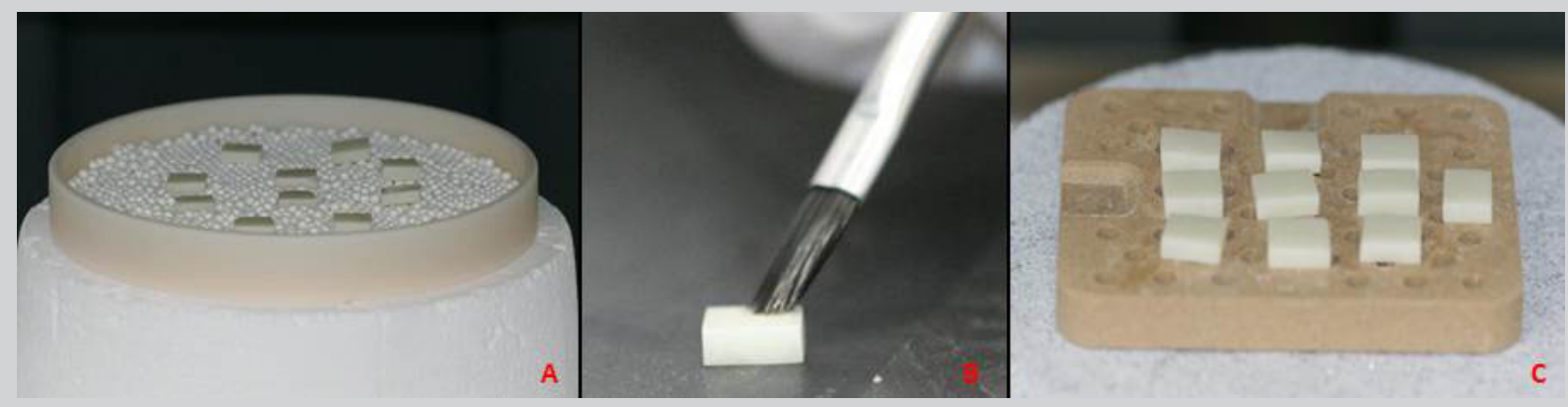

Figura 1 A-C - A) Blocos de zircônia em forno Zyrcomat (Vita Zahnfabrik) após sinterização; B) Aplicação de glaze com pincel, segundo recomendações do fabricante; C) amostras glazeadas após ciclo de queima em forno Vacumat 6000MP (Vita Zahnfabrik). 
de $20 \times$ e em uma área de $301,3 \times 229,2 \mu \mathrm{m}$. Os valores de rugosidade foram obtidos em $\mu \mathrm{m}(\mathrm{Ra})$.

\section{Análise dos resultados}

Os resultados foram analisados por Análise de Variância (ANOVA) dois-fatores e Teste de Tukey (á=5\%). O teste de Kolmogorov-Smirnov demonstrou que os dados seguiram uma distribuição normal.

\section{RESULTADOS}

O fator "concentração do ácido fluorídrico" não foi significante estatisticamente. No entanto, o fator "tempo de condicionamento" foi significante (Tabela 2). Além disso, a interação entre os fatores em estudo (concentração do ácido fluorídrico e tempo de condicionamento) foi estatisticamente significante $(p=0,00)$ (ANOVA) (Tabela 2 e Figura 2).

* significância estatística $(p<0,05)$

Os valores de rugosidade média ( $\mathrm{Ra})( \pm$ desvio-padrão) de todos os grupos estão apresentados na tabela 3 . Os protocolos de condicionamento dos grupos HF10/20 e HF5/90 obtiveram os maiores valores de rugosidade média. Os grupos HF5/60, HF10/60 e HF10/90 obtiveram valores de rugosidade média estatisticamente semelhantes e menores que os grupos supracitados. O grupo HF5/ 20 obteve valores estatisticamente semelhantes tanto aos grupos de maiores valores de rugosidade média quanto aos de menores valores (tabela 3 ). As imagens em 3D resultantes da análise em perfilômetro digital estão apresentadas na figura 3 .

A hipótese testada foi aceita, uma vez que os protocolos de condicionamento resultaram em valores de rugosidade diferentes.

\begin{tabular}{|c|c|c|c|c|c|c|}
\hline Fator & $\mathrm{ql}$ & Seq SQ & Adi SQ & Adi QM & $\mathrm{F}$ & $P$ \\
\hline $\begin{array}{l}\text { Concentração do ácido } \\
\text { fluorídrico }\end{array}$ & 1 & 0,4914 & 0,4914 & 0,4914 & 2,15 & 0,149 \\
\hline $\begin{array}{c}\text { Tempo de } \\
\text { condicionamento }\end{array}$ & 2 & 2,3617 & 2,3617 & 1,1809 & 5,16 & $0,009^{*}$ \\
\hline $\begin{array}{l}\text { Concentração do ácido } \\
\text { fluorídrico x Tempo de } \\
\text { condicionamento }\end{array}$ & 2 & 7,1884 & 7,1884 & 3,5942 & 15,70 & $0,000^{*}$ \\
\hline $\begin{array}{l}\text { Erro } \\
\text { Total }\end{array}$ & $\begin{array}{l}54 \\
59\end{array}$ & $\begin{array}{l}12,3626 \\
22.4041\end{array}$ & 12,3626 & 0,2289 & & \\
\hline
\end{tabular}

*siqnificância estatística $(\mathrm{p}<0.05)$

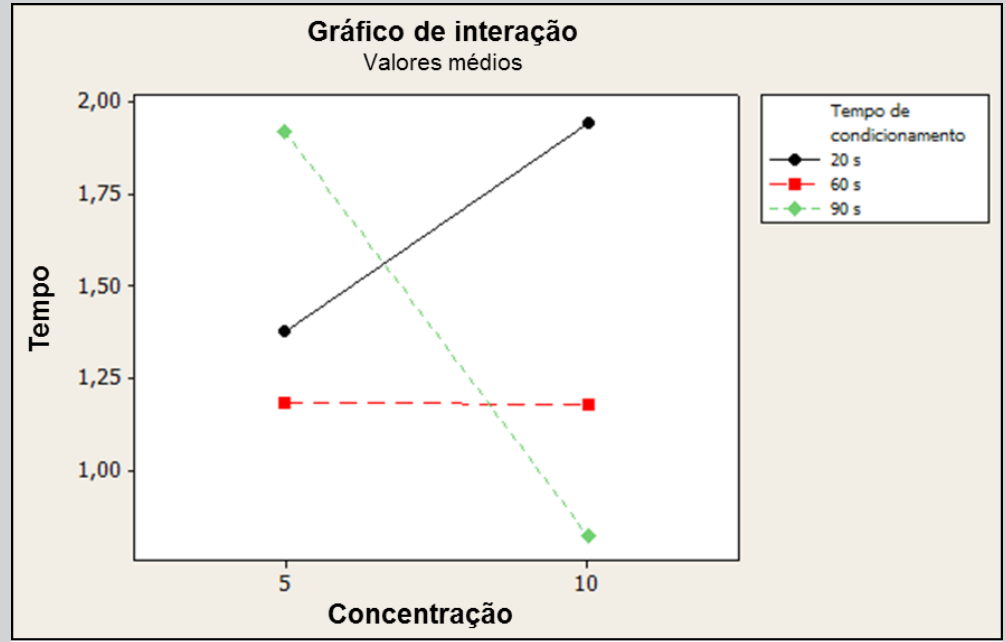

Figura 2 - Gráfico de interação mostrando como o fator tempo de condicionamento atuou nas duas concentrações de ácido fluorídrico utilizadas. 


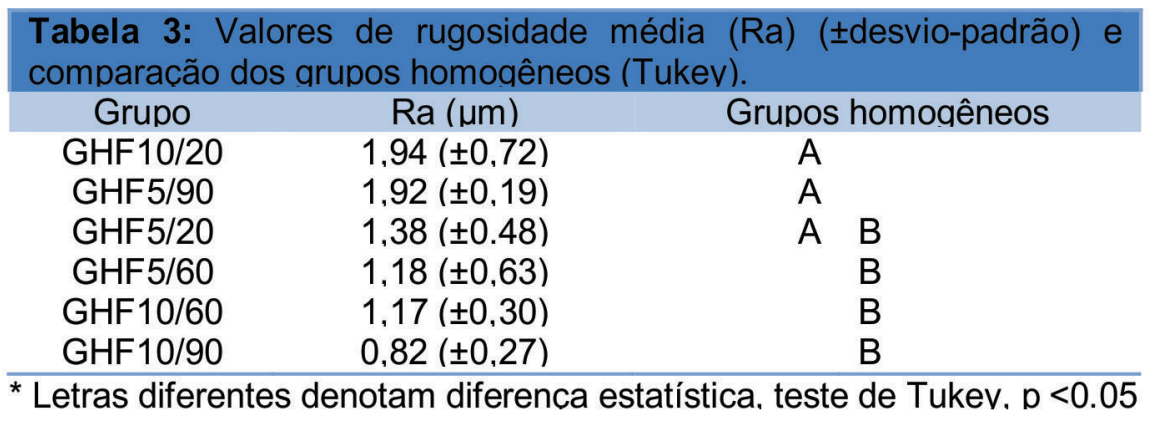

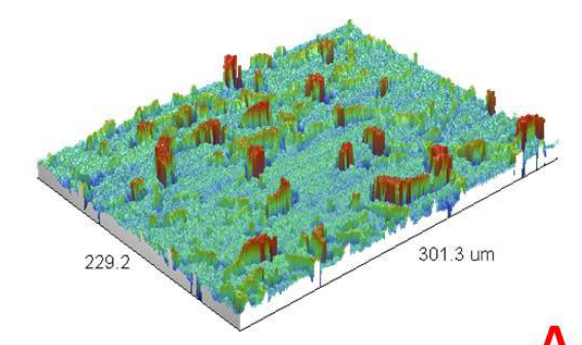

A

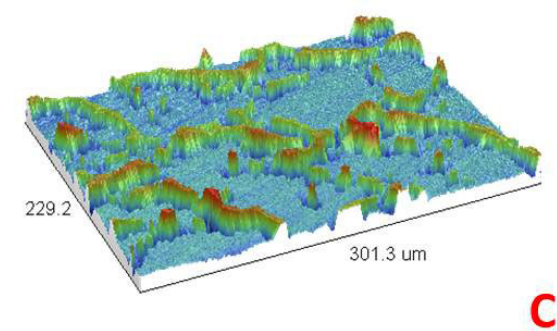

C

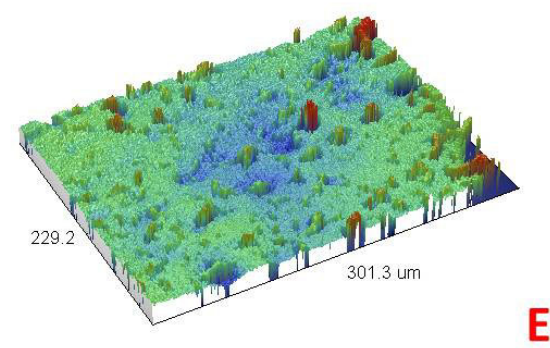

E

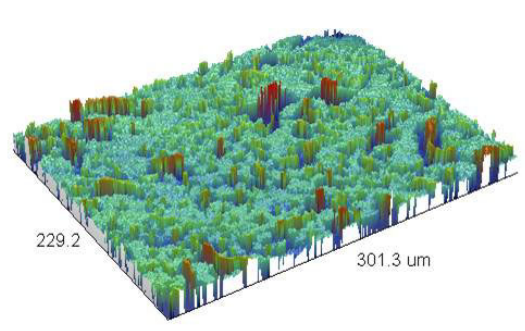

B
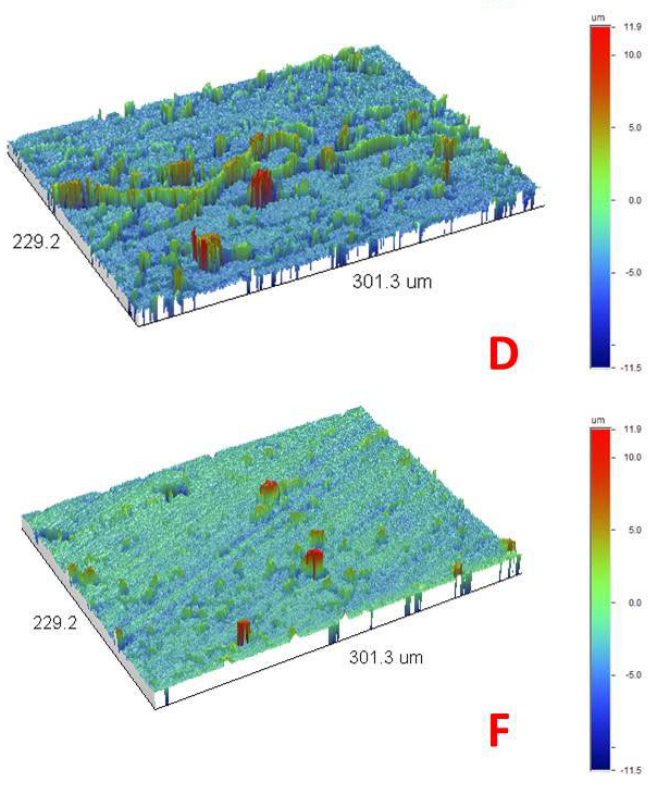

Figura 3 A-E - Imagens 3D obtidas pelo perfilômetro óptico digital: A) Grupo GHF5/20; B) Grupo GHF5/60; C) Grupo GHF5/90; D) Grupo GHF10/20; E) Grupo GHF10/60; F) Grupo GHF10/90.

\section{DISCUSSÃO}

Nos tratamentos protéticos reabilitadores, a zircônia tem sido o material cerâmico de escolha quando se deseja associar estética à resistência mecânica ${ }^{20}$. Entretanto, uma das limitações das restaurações de zircônia está relacionada a sua adesividade ao cimento resinoso, por se tratar de cerâmicas ácido resistentes. Dessa forma, diversos estudos têm sido realizados para avaliar a 
resistência de união entre materiais cerâmicos a base de zircônia e cimentos resinosos ${ }^{21,22,23}$.

Diversos tratamentos de superfície têm sido propostos na literatura para melhorar a adesão entre a zircônia e o cimento resinoso, como: jateamento com partículas de óxido de alumínio ${ }^{12}$, jateamento com partículas de óxido de alumínio revestidas por sílica ${ }^{24,25}$, aplicação de primers específicos para zircônia ${ }^{26}$, deposição de filmes finos de sílica ${ }^{9} \mathrm{e}$ utilização de cimentos resinosos contendo monômeros fosfatados (MDP) em sua composição ${ }^{10,11}$.

Com relação ao jateamento, a literatura ${ }^{27}$ tem relatado que o uso de partículas de alumina $(50 \mu \mathrm{m})$ ou de partículas de alumina revestida por sílica (30 $\mu \mathrm{m})$ na superfície cerâmica, seguido de cimentação com um cimento resinoso dual e submetidos ao ensaio de cisalhamento exibem bons valores de resistência adesiva, que diminuem quando submetido a termociclagem. Já outros estudos ${ }^{28,29}$ tem observado que o abrasionamento não teve um efeito positivo na melhoria da união do cimento resinoso a zircônia. Entretanto, sabe-se que o jateamento tem sido criticado por possivelmente levar a fragilização da zircônia ${ }^{14}$. Isso tem levado a práticas de diferentes técnicas no intuito de promover valores de resistência de união semelhantes ou maiores ao tratamento com jateamento, mas que possa evitar esses possíveis efeitos deletérios.

Recentemente, uma proposta de modificação da superfície da zircônia por meio de aplicação de uma camada vítrea tem sido avaliada ${ }^{6,7,16,19,30}$. Esta técnica baseia-se na modificação da superfície de cimentação, pela aplicação de uma camada de glaze (cerâmica vítrea), que pode ser degradada mais facilmente pela ação do ácido fluorídrico.

Por ser a rugosidade superficial considerada um outro importante fator para a adesão, já que aumenta a área de superfície, melhora a molhabilidade pela redução na tensão da superfície e cria retenção micromecânica ${ }^{30}$, o presente estudo alterou a superfície da zircônia através da aplicação do glaze e avaliou o efeito de diferentes protocolos de condicionamento na rugosidade superficial de uma zircônia glazeada. O condicionamento com ácido fluorídrico da superfície de cimentação da zircônia glazeada e posterior silanização, parece ser um método efetivo para se melhorar a adesão de cimentos resinosos à zircônia.

Um estudo recente ${ }^{30}$ observou que a irradiação da zircônia com laser Nd: YAG resultaram tanto em aumento da rugosidade superficial, e da resistência de união do cimento resinoso. Já a zircônia glazeada e condicionada com ácido fluorídrico $(0,67 \mu \mathrm{m} ; 4,99 \mathrm{MPa})$, o grupo controle $(0,16 \mu \mathrm{m} ; 3,73 \mathrm{MPa})$ e a zircônia abrasionada $(0,19$ $\mu \mathrm{m} ; 4,26 \mathrm{MPa}$ ) tiveram comportamento semelhante quanto a esses valores.

Entretanto, estudo recente ${ }^{16}$ observou que a aplicação de glaze superficial seguido de condicionamento com ácido fluorídrico e silanização na zircônia levou a uma maior resistência de união. Os valores de rugosidade superficial e resistência de união do grupo controle $(0,14 \mu \mathrm{m} ; 2,3 \mathrm{MPa})$ variaram dos grupos: glazeado com spray e condicionado com ácido fluorídrico a 9\% (3.37 $\mu \mathrm{m}$; 13,3 MPa); glazeado com spray e silicatização $(0,41 \mu \mathrm{m} ; 9,2 \mathrm{Mpa}) ;$ glazeado com pincel e condicionado com ácido fluorídrico a $9^{\circ} 8(3,79 \mu \mathrm{m}$; $17,8 \mathrm{Mpa})$; e glazeado com pincel e condicionado com ácido fluorídrico a $9 \%(0,4 \mu \mathrm{m} ; 12,5 \mathrm{Mpa})$

Resultados divergentes ${ }^{7}$ foram reportados pelos autores, os quais observaram que a aplicação da camada de glaze melhorou significativamente a resistência de união no grupo silano / adesivo / Variolink (9MPa) em comparação ao grupo não glazedo (2,4 MPa) em condições secas. Já para o grupo zirconia primer / Multilink, o grupo glazeado mostrou resistência de união significativamente mais baixas $(4,9 \mathrm{MPa})$ em relação ao grupo glazeado (10,9 Mpa), também em condições secas.

A efetividade do glaze na melhoria da resistência de união, também tem sido avaliada pela literatura ${ }^{19}$. Esses autores observaram tanto o jateamento com $\mathrm{Al}_{2} \mathrm{O}_{3}$ de 50 e $100 \mu \mathrm{m}$ promovem efeitos similares na resistência adesiva entre cerâmica e cimento resinoso, porém a adição do glaze na superfície de cimentação da zircônia aumenta a resistência adesiva ao cimento resinoso. Os grupos que utilizaram o glaze em associação com ácido fluorídrico, sem silano (20,75 MPa) e 
com silano (25,17 MPa), ou jateamento, sem silano $(17,45 \mathrm{MPa})$ e com silano $(18,41)$ mostraram uma resistência de união superior aos grupos que apenas jatearam com partículas de óxido de alumínio 50 e $110 \mu \mathrm{m}$. Já outro estudo ${ }^{6}$ revelou que a aplicação de um glaze contendo uma maior fase de dissilicato de lítio pode ser um passo importante para aumentar a união da zircônia ao cimento resinoso.

Neste estudo, a hipótese inicial de que os protocolos de condicionamento resultariam em valores de rugosidade diferentes foi confirmada. Os resultados deste estudo mostraram que os tratamentos de superfície alteraram significativamente a rugosidade da cerâmica. Observou-se interação estatisticamente significante entre os fatores concentração do ácido fluorídrico (2 níveis $5 \%$ e $10 \%$ ) e tempo de condicionamento ( 3 níveis - 20 s, 60 s e 90 s). Na concentração do ácido fluorídrico de $10 \%$, tanto no tempo de condicionamento de 60 quanto de 90 segundos, menores valores de rugosidade foram obtidos. É possível afirmar que a elevada concentração do ácido fluorídrico (10\%), associada a maiores tempos de condicionamento ( 60 s e $90 \mathrm{~s}$ ), teria removido a camada vítrea criada, deixando a superfície menos rugosa. A superfície parece estar bem uniforme, ou seja, sem irregularidades, principalmente após o maior tempo de condicionamento (90 s). Entretanto, esta concentração de ácido fluorídrico (10\%) com um tempo menor de condicionamento (20 s) promoveu maiores valores da rugosidade.

Nos grupos em que se utilizou ácido fluorídrico na concentração de $5 \%$, os valores de rugosidade média encontrada foram mais altos para os tempos de 90 e 20 segundos e menores para o tempo de 60 segundos. Isso poderia ser explicado pela possibilidade de a camada de glaze já possuir uma rugosidade acentuada, pois a aplicação feita com pincel não resulta em camada uniforme ${ }^{16}$. Além disso, o solvente presente no glaze evapora durante o ciclo de queima, gerando uma superfície irregular. O tempo de 20 segundos com ácido fluorídrico a 5 $\%$ não levaria então à dissolução seletiva da camada vítrea. Já o tempo de condicionamento de 60 segundos faria uma remoção das irregularidades, que seriam então redefinidas após condicionamento por 90 segundos. A superfície após condicionamento por 60 segundos com ácido $5 \%$ é bem mais uniforme do que as outras superfícies do mesmo grupo.

A utilização do glaze aplicado sobre superfícies resistentes a condicionamento com ácido fluorídrico deve ser melhor compreendida em estudos posteriores de resistência de união, uma vez que outros fatores podem estar envolvidos nesse processo de adesão à zircônia. Entretanto, estudos clínicos randomizados de adesão à zircônia glazeada com e concentração do ácido fluorídrico devem ser realizados para confirmar os achados laboratoriais.

\section{CONCLUSÃO}

Pode-se concluir que os protocolos de condicionamento da zircônia glazeada utilizados resultaram em valores de rugosidade média superficial diferentes, pois o ácido fluorídrico (10\%) em maior concentração, associado a um menor tempo de condicionamento (20 s), promoveu maior rugosidade superficial da zircônia glazeada.

\section{REFERÊNCIAS}

1. Doi M, Yoshida K, Atsuta M, Sawase $T$. Influence of pre-treatments on flexural strength of zirconia and debonding crack-initiation strength of veneered zirconia. J Adhes Dent. 2011;13(1):79-84.

2. Everson P, Addison O, Palin WM, Burke FJ. Improved bonding of zirconia substructures to resin using a "glaze-on" technique. J Dent. 2012;40(4):347-51.

3. Kosmac T, Oblak C, Jevnikar P, Funduk N, Marion L. The effect of surface grinding and sandblasting on flexural strength and reliability of Y-TZP zirconia ceramic. Dent Mater. 1999;15(6):426-33.

4. Molin MK, Karlsson SL. Five-year clinical prospective evaluation of zirconia-based Denzir 3-unit FPDs. Int J Prosthodont 2008; 21(3): 223-227.

5. Tinschert J, Schulze KA, Natt G, Latzke P, Heussen N, Spiekermann H. Clinical behavior of zirconia-based fixed partial dentures made of DC-Zirkon: 3-year results. Int J Prosthodont. 2008;21(3):217-22.

6. Ntala P, Chen X, Niggli J, Cattell M.Development and testing of multi-phase glazes for adhesive bonding to zirconia substrates. J Dent. 2010;38(10):773-81. 
7. Cura C, Özcan M, Isik G, Saracoglu A. Comparison of alternative adhesive cementation concepts for zirconia ceramic: glaze layer vs zirconia primer. J Adhes Dent. 2012;14(1):75-82.

8. Ozcan M, Vallittu PK. Effect of surface conditioning methods on the bond strength of luting cement to ceramics. Dent Mater. 2003;19(8):725-31.

9. Queiroz JR, Massi M, Nogueira L Jr, Sobrinho AS, Bottino MA, Ozcan M. Silica-based nano-coating on zirconia surfaces using reactive magnetron sputtering: effect on chemical adhesion of resin cements. J Adhes Dent. 2013;15(2):151-9

10. Wolfart M, Lehmann F, Wolfart S, Kern M. Durability of the resin bond strength to zirconia ceramic after using different surface conditioning methods. Dent Mater. 2007;23(1):45-50

11. Akgungor G, Sen D, Aydin M. Influence of different surface treatments on the short-term bond strength and durability between a zirconia post and a composite resin core material. J Prosthet Dent. 2008;99(5):38899.

12. Blatz MB, Chiche G, Holst S, Sadan A. Influence of surface treatment and simulated aging on bond strengths of luting agents to zirconia. Quintessence Int. 2007 38(9): 745-753.

13. Guazzato M, Quach L, Albakry M, Swain MV. Influence of surface and heat treatments on the flexural strength of Y-TZP dental ceramic. J Dent. 2005;33(1):9-18.

14. Zhang $Y$, Lawn BR, Malament KA, Van Thompson $P$, Rekow ED. Damage accumulation and fatigue life of particle-abraded ceramics. Int J Prosthodont. 2006;19(5):442-8

15. Cattell MJ, Chadwick TC, Knowles JC, Clarke RL. The development and testing of glaze materials for application to the fit surface of dental ceramic restorations. Dent Mater 2009; 25(4): 431-441.

16. Vanderlei A, Bottino MA, Valandro LF. Evaluation of resin bond strength to yttria-stabilized tetragonal zirconia and framework marginal fit: comparison of different surface conditionings. Oper Dent. 2014;39(1):50-63.

17. Brentel AS, Ozcan M, Valandro LF, Alarca LG, Amaral R, Bottino MA. Microtensile bond strength of a resin cement to feldpathic ceramic after different etching and silanization regimens in dry and aged conditions. Dent Mater. 2007; 23(11): 1323-1331.

18. Phoenix RD, Shen C. Characterization of treated porcelain surfaces via dynamic contact angle analysis. Int J Prosthodont. 1995;8(2):187-94

19. Valentino TA, Borges GA, Borges LH, Platt JA, CorrerSobrinho L. Influence of glazed zirconia on dual-cure luting agent bond strength. Oper Dent 2012; 37(2): 181 187.

20. Souza ROAO, MIYASHITAEM. Zircônia na Odontologia: Vantagens e possíveis limitações. REABILITAÇÃO ORAL - PREVISIBILIDADE E LONGEVIDADE - Livro Oficial Do $11^{\circ} \mathrm{CIOMIG}$ 2011. 1. Belo Horizonte - MG: NAPOLEAO 2011. p. 531-582.
21. Aboushelib MN, Mirmohamadi H, Matinlinna JP, Kukk E, Ounsi HF, Salameh Z. Innovations in bonding to zirconiabased materials. Part II: Focusing on chemical interactions. Dent Mater. 2009;25(8): 989-993.

22. Matinlinna JP, Lassila LV. Enhanced resin-composite bonding to zirconia framework after pretreatment with selected silane monomers. Dent Mater. 2011;27(3):27380.

23. May LG, Passos SP, Capelli DB, Ozcan M, Bottino MA, Valandro LF. Effect of silica coating combined to a MDPbased primer on the resin bond to Y-TZP ceramic. J Biomed Mater Res B Appl Biomater. 2010;95(1):69-74.

24. Ozcan M, Nijhuis $H$, Valandro LF. Effect of various surface conditioning methods on the adhesion of dualcure resin cement with MDP functional monomer to zirconia after thermal aging. Dent Mater J. 2008;27(1):99-104.

25. de Castro HL, Corazza PH, Paes-Júnior T de A, Della Bona. A Influence of Y-TZP ceramic treatment and different resin cements on bond strength to dentin. Dent Mater 2012; 28(11): 1191-1197.

26. Kobes KG, Vandewalle KS. Bond strength of resin cements to zirconia conditioned with primers. Gen Dent. 2013; 61(6): 73-76.

27. Passos SP, Valandro LF, Bottino MA, Santos MJ, Santos GC Jr. Shear bond strength of resin cement bonded to alumina ceramic after treatment by aluminum oxide sandblasting or silica coating. J Prosthodont. 2011;20(7):561-5.

28. Atsu SS, Kilicarslan MA, Kucukesmen HC, Aka PS. Effect of zirconium-oxide ceramic surface treatments on the bond strength to adhesive resin. J Prosthet Dent. 2006;95(6):430-6.

29. Dérand $P$, Dérand $T$. Bond strength of luting cements to zirconium oxide ceramics. Int $\mathrm{J}$ Prosthodont. 2000;13(2):131-5.

30. Usumez A, Hamdemirci N, Koroglu BY, Simsek I, Parlar $\mathrm{O}$, Sari T. Bond strength of resin cement to zirconia ceramic with different surface treatments. Lasers Med Sci. 2013;28(1):259-66.

\section{Correspondência}

Rodrigo Othávio de Assunção e Souza

Av. Salgado Filho, 1787. Lagoa Nova, Natal/RN, Brasil. CEP: 59056-000

Email: rodrigoothavio@gmail.com 